\title{
Privacidad en redes sociales: análisis de los riesgos de auto-representación digital de adolescentes españoles
}

\section{Privacy in social networks: analysis of the spanish teenagers digital self-presentation risks}

María José Hernández-Serrano. Universidad de Salamanca. España.

mjhs@usal.es

$[\mathrm{CV}] \bigcirc \mathrm{G} \mathrm{R}^{\mathrm{a}} \mathrm{O}$

Paula Renés-Arellano. Universidad de Cantabria. España.

renesp@unican.es

$[\mathrm{CV}]\left(\mathcal{G} \mathbb{R}^{\circ} \mathrm{O}\right.$

Rosalynn Argelia Campos Ortuño. Universidad de Salamanca, de la Universidad Internacional de la Rioja y Nebrija. España.

rosecampos@usal.es

$[\mathrm{CV}](\mathrm{G})$

Belén González-Larrea. Universidad de Salamanca. España.

belengonzalezlarrea@usal.es

$[\mathrm{CV}]$ (1) $\mathrm{R}^{\mathrm{a}}$

Este trabajo se enmarca en los proyectos, CONECT-ID. La identidad hiperconectada de la juventud y su percepción del tiempo en el ocio digital». Ministerio de Ciencia, Innovación y Universidades. Referencia: PGC2018-097884-B-I00 (2019-2021); con el apoyo del Proyecto I+D "Youtubers $e$ Instagrammers: la competencia mediática en los prosumidores emergentes", Referencia: RTI2018093303-B-I00 (2020-2022) de la Red Alfamed' (Red Euroamericana de Investigadores), financiados por la Agencia Estatal de Investigación del Ministerio de Ciencia, Innovación y Universidades de España y el Fondo Europeo de Desarrollo Regional.

Cómo citar este artículo / Referencia normalizada

Hernández-Serrano, Ma J., Renés-Arellano, P., Campos Ortuño, R. y González-Larrea, B. (2021). Privacidad en redes sociales: análisis de los riesgos de auto-representación digital de adolescentes españoles. Revista Latina de Comunicación Social, 79, 133-154. https://www.doi.org/10.4185/RLCS-2021-1528

\section{RESUMEN}

Introducción: Las competencias digitales son referentes para la formación de los adolescentes ante los nuevos procesos de digitalización, en especial sobre el uso de redes sociales donde consumen y producen información personal a través de diferentes prácticas de auto-representación digital. Estas prácticas y los riesgos que suponen para la privacidad de los menores son descritas en este trabajo a partir de la revisión de estudios recientes en torno al tema y de los datos de una muestra de adolescentes españoles $(n=2066)$ de entre 12 y 18 años. Metodología: La metodología ha sido cuantitativa empleando un cuestionario con escalas tipo Likert, que cumple con criterios de validación de expertos y la fiabilidad, respondido de forma anónima por los menores. Resultados: 
Entre los resultados destacan prácticas similares en tres de las redes sociales más usadas por los adolescentes (WhatsApp, Instagram y Spotify), asociadas al uso de fotos, nombre y ubicación real, así como al no filtrado de audiencias, siendo conscientes los menores de que compartir información personal supone riesgos para su privacidad y habiendo recibido formación al respecto. Discusión y Conclusiones: Finalmente, se concluye que la formación en conductas protectoras debe reforzarse dentro de las competencias digitales básicas, para que los menores aprendan a interaccionar de forma segura en las redes sociales, con responsabilidad y cuidando su privacidad y auto-representación digital.

PALABRAS CLAVE: competencia digital; adolescentes; auto-representación digital; redes sociales; riesgos de Internet; uso responsable de redes sociales; habilidades digitales.

\begin{abstract}
Introduction: Digital competencies are a reference for the training of adolescents in the face of new digitalization processes, especially in the use of social networks where they consume and produce personal information through different digital self-representation practices. These practices and the risks they pose to the privacy of minors are described in this paper based on a review of recent studies and data from a sample of Spanish adolescents $(n=2066)$ aged between 12 and 18 years. Methodology: The methodology was quantitative, using a Likert-type scale questionnaire that complies with expert validation criteria and reliability, answered anonymously by the participants. Results: Among the results, similar practices stand out in three of the social networks most used by adolescents (WhatsApp, Instagram and Spotify), associated with the use of personal photos, real name and location, as well as the non-filtering of audiences, being adolescents aware that sharing personal information poses risks to their privacy and having received training in this regard. Discussion and Conclusion: Finally, it is concluded that training in protective behaviors should be reinforced into basic digital skills, so that adolescents learn to interact safely on social networks, with responsibility and taking care of their privacy and digital self-representation.
\end{abstract}

KEYWORDS: digital competence; adolescents; digital self-representation; social networks; internet risks; responsible use of social networks; digital skills.

\title{
CONTENIDO
}

1. Introducción. 1.1. Las competencias digitales y la privacidad online 1.2. Auto-representación digital y riesgos para la privacidad de los menores 2. Objetivos. 3. Metodología. 4. Resultados. 5. Discusión y Conclusiones. 6. Bibliografía 7. Currículum Vitae

\section{Introducción}

\subsection{Las competencias digitales y la privacidad online}

Las competencias digitales, entendidas como el conjunto de capacidades y habilidades que permiten a las personas ser responsables y críticas ante los medios, ser capaces de buscar, procesar, comunicar, crear y difundir información por medio de las tecnologías (Ferrés y Piscitelli, 2012; Pérez-Rodríguez y Delgado-Ponce, 2012) se han convertido en competencias fundamentales en la formación de la ciudadanía (Atchoarena et al., 2017; González et al., 2018) e imprescindibles para el aprendizaje permanente (Casillas-Martín et al., 2020), porque permiten a las personas adaptarse al ecosistema mediático actual, siendo responsables y reflexivas en el uso de las TIC (Tejada y Pozos, 2018).

Las competencias digitales son clave para el desarrollo y la inclusión de todos los colectivos, si bien, son un referente para la formación de los menores y jóvenes, para quienes los procesos de 
digitalización están ofreciendo mayores posibilidades de acceso a cualquier contenido digital, tanto público como privado (Quintas-Froufe y González-Neira, 2021). Los menores y jóvenes se convierten en los protagonistas indiscutibles de los canales de comunicación digital (García, et al., 2013; Núñez y Ruta, 2019; Seo et al., 2014) donde cada vez de forma más temprana comparten, diseñan o generan contenidos e informaciones sobre su vida cotidiana. Sin embargo, ni la facilidad, ni el rápido manejo de las tecnologías garantizan que sean competentes o que puedan ser capaces de evitar riesgos digitales (Aguaded et al., 2007). Y aquí se sitúa el contexto de esta investigación, advirtiendo que cuando los menores publican, consumen o interaccionan con información digital exponen de forma continuada su identidad (Livingstone et al., 2010), mostrando en las redes información que les define y les enfrenta a riesgos para su privacidad (Peñalva et al., 2018), tanto los asociados al uso de las redes sociales, de los que a veces no son conscientes (De la Villa Moral y Fernández, 2019 ), como otros que pueden asociarse al consumo de información procedente de páginas no fiables (Siddiqui y Sigh, 2016), al uso inapropiado del lenguaje o a las adicciones y exceso de utilización de las tecnologías (Cabero et al., 2020; Pontes et al., 2015).

Ante los riesgos que amenazan la privacidad de los menores, desde diferentes organismos internacionales se insta a favorecer y promover las competencias digitales entre los menores, como son la Agenda 2030 (2020) o el Manifiesto de Infancia y Adolescencia de UNICEF (2020). En concreto, la Comisión Europea publicó el Marco Europeo para la Competencia Digital de los Ciudadanos (Ferrari, 2013), y otro marco similar para el contexto educativo DIGCompEdu (Redecker, 2017) en los que se describe cómo empoderar y formar a los menores y jóvenes en las capacidades necesarias para interactuar o compartir contenido en la esfera digital, gestionar de forma adecuada la identidad digital o promover comportamientos adecuados en las redes sociales, entre algunas de las habilidades más relevantes. Estos marcos son guía a nivel nacional o local de diferentes programas y acciones educativas que cada vez son más frecuentes en los entornos escolares y extraescolares para la prevención y protección ante los riesgos digitales (McDougall et al., 2018).

Considerada como competencia digital clave para los menores y jóvenes, la protección de la privacidad en los entornos digitales debe ser una prioridad formativa desde diferentes instancias educativas, para que pueda convertirse no sólo en una habilidad, sino también en un valor a potenciar. Tomar conciencia de su importancia requiere analizar conjuntamente cuáles son las prácticas de auto-representación digital más frecuentes de los menores y cómo dichas prácticas les exponen ante riesgos para su privacidad. En esta línea, expondremos en este trabajo un estado de la cuestión a partir de estudios recientes y presentaremos los resultados de una investigación nacional, en el contexto español, sobre los riesgos asociados a prácticas de auto-representación digital de adolescentes en redes sociales.

\subsection{Auto-representación digital y riesgos para la privacidad de los menores}

La digitalidad ha supuesto importantes implicaciones para el desarrollo de la gestión de la identidad y la privacidad, con un impacto aún más evidente para los menores, quienes forman parte de una cultura que les insta a estar y participar online, tanto para redefinirse como para socializar. El uso de redes sociales ha aumentado en el último año (Kemp, 2021), especialmente entre los menores, destacando el mayor uso de Instagram (Statista, 2021). El empleo de estas redes se ha generalizado a nivel intergeneracional y transcultural, empezando a normalizarse la publicación de contenido de auto-representación cada vez más visual (Gioia et al., 2021), aumentando los riesgos de privacidad y vigilancia (Adorjan y Ricciardelli, 2020) y, con ellos, los nuevos ámbitos de interés para la alfabetización mediática y digital. 
Entre las diferentes dimensiones de la privacidad afectadas por la digitalidad (Stuart et al., 2019), asistimos a un momento de redefinición de la denominada "privacidad expresiva" (DeCew, 1997), relacionada con la expresión de uno mismo y de su identidad, que tradicionalmente venía asociándose a prácticas y valores de protección de los aspectos íntimos, pero que en la actualidad se ve reinterpretada y es explotada públicamente (Richardson, 2017). En referencia a los primeros espacios digitales -redes más textuales y no tan visuales ni interactivas como las que se ofrecen en la actualidad- Baym y Boyd (2012) ya venían advirtiendo una dilución entre las fronteras de lo público y lo privado, el control y la libertad, o lo virtual y lo real, para explicar que los usuarios estaban empezando a gestionar su "yo privado" en las fronteras de lo público, considerando la repercusión que sus prácticas tendrían en las audiencias a las que se querían dirigir. Hoy tenemos redes y plataformas que permiten mayores posibilidades de expresión y representación, con mecanismos más sofisticados para delimitar qué contenidos publicados pueden ser vistos por distintas redes de contactos privados y cuáles prefieren extenderse al ámbito público. Es interesante el fenómeno que podríamos denominar "confusión de control de privacidad", por el cual las audiencias se ven empoderadas ante la posibilidad de poder discriminar lo que se quiere publicar para diferentes audiencias, provocando una falsa sensación de protección o anonimato. En el caso de los adolescentes, los estudios confirman que están más preocupados por limitar el acceso de sus contenidos personales a figuras de autoridad, como padres y profesores (Torrecillas et al., 2020), que por protegerse ante la publicidad no deseada (Jeong y Coyle, 2014) o las consecuencias de la vulneración de su información personal, tanto para ellos, como para sus familiares, tanto pensando en su momento actual como a futuro (Reid y Boyer, 2013).

En la misma línea, podríamos considerar con Rodríguez et al. (2017) la idea de "privacidad aumentada", como resultado del reconocimiento social y el crecimiento emocional que se ven multiplicados por la difusión online, incrementando así el deseo de continuar publicando información personal. Hay que tener en cuenta que el hecho de compartir información personal es un aspecto diferencial, a veces determinante, en las posibilidades de socialización (Crescenzi et al., 2013) y que, a nivel psicológico, se ha explicado suficientemente que la auto-revelación forma parte del proceso social que permite, o es usado, para formar y mantener relaciones de cercanía o confianza (Reisenzein et al., 2012); en otras palabras, divulgar información que nos define también nos acerca o nos hace sentir cerca de otros (Davis, 2013). Y todo ello, sitúa al usuario de redes sociales ante la decisión de optar por proteger su privacidad o fomentar su sociabilidad y reconocimiento, prefiriendo en muchas ocasiones los beneficios sociales de la publicación de su información privada.

Este es sin duda uno de los retos más apremiantes con los que nos encontramos en la actualidad, dirigido a visibilizar la necesidad de que la ciudadanía conozca los riesgos que suponen algunas de sus prácticas de auto-representación digital y aprenda a proteger su información personal en redes sociales. Si cabe, es una necesidad aún más urgente para los menores quienes, por sus necesidades y desarrollo evolutivo, acaban sacrificando su protección, para anteponer la popularidad y el prestigio social que les ofrecen las redes sociales y las interacciones digitales. Entre los múltiples beneficios que los adolescentes encuentran en las redes sociales para su auto-representación podríamos situar al menos dos variables explicativas de los riesgos crecientes, que además fomentan un uso intensivo y poco seguro: por un lado, la ampliación de oportunidades, es decir, el hecho de que las plataformas y servicios digitales que ofrecen auto-representación e interacción online con otros usuarios han expandido las posibilidades y formas de comunicación offline, siendo estas nuevas formas mayormente aprovechadas, renegociadas y reinventadas por los jóvenes (Rodríguez et al., 2017; Wood et al., 2016). De esta forma, la autoaceptación depende de las múltiples formas de expresión y representación y de cómo se recibe el feedback, o se valida socialmente lo que se publica online, como confirman diferentes estudios recientes (Aldrin, 2019; Barth y De Jong, 2017; Bell, 2019; Cipolletta et al., 2020; Spiller, 2020). Y por otro lado, a un mayor control de lo que se denomina 
"gestión de la impresión", es decir, el poder controlar la impresión online que se desea causar en un público objetivo o deseado (Bell, 2019), no sólo porque se puede delimitar a los destinatarios de lo que se publica (Scott y Fullwood, 2020), sino porque existe mayor posibilidad de congruencia al poder continuar una historia digital de auto-representación, para que no resulte disonante con la identidad mostrada ya por el individuo (Leong, 2016), a todo ello se añaden las ventajas de la asincronía y del ensayo previo a la publicación (Ditchfield, 2020; Gioia et al., 2021) que permiten la mejora y la modificación de lo que se desea publicar para representarse, frente a la instantaneidad del mundo offline.

Haciendo balance a estas posibilidades se sitúan los riesgos de estas prácticas digitales, con atención a los que han sido especialmente estudiados para el colectivo de menores y adolescentes. Entre los riesgos, Aldrin (2019) ha documentado la importancia que tiene el nombre de usuario que se elige para la auto-representación, que es público, donde los menores pueden estar revelando sus intereses personales, su edad, género, sexualidad, apariencia física, etnicidad, u orientaciones culturales y religiosas. Doster (2013) ha expuesto cómo las fotos que publican los adolescentes no sólo revelan la apariencia física o los comportamientos, sino que las posesiones y los objetos también ofrecen información del estatus social, como han confirmado otros estudios (Hong et al., 2020). También los vídeos y los fenómenos "challenge" fomentan la comparación social de habilidades (Yang et al., 2018) generando una identidad digital irreal o exagerada. Crescenzi et al., (2013) han expuesto que, con bastante regularidad y sin ocultarse, los adolescentes hacen pública su ubicación en tiempo real, sea por geolocalización, o indirectamente a través de mensajes o fotos de lugares, lo que conlleva otros riesgos que pueden extenderse al mundo offline, o exponerse ante otros ciber-riesgos como el grooming, la sextorsion o el ciberbullying (Montiel et al., 2016); Schwartz y Halegoua (2015) han estudiado también cómo la localización compartida genera asociaciones de usuarios a lugares, pudiendo llevar a conclusiones erróneas o no relacionadas con el sujeto, sus preferencias y lo que define a esos lugares. Bell (2019) ha resaltado también la ambigüedad de la característica efímera que define a algunos espacios digitales, que son interpretados así, cuando en realidad la mayor parte de la información que se publica online no desaparece y permanece accesible en el tiempo, haciendo que la auto-representación pueda ser contradictoria a largo plazo, o interfiriendo en la identidad y los roles que se alcancen a futuro. Además, el hecho de que los servicios online y las distintas redes se vinculen entre sí hace que cada vez sea más difícil separar la información personal que se publica en diferentes espacios, accediéndose a diferentes tipos de información de auto-representación (Emanuel y Stanton, 2014), que ya no está dispersa, ni limitada, aunque el usuario la hubiera preseleccionado para distintas audiencias.

Estos riesgos no son desconocidos por el usuario, es decir, los estudios en torno al tema ponen de manifiesto que entre el colectivo de menores y jóvenes existe preocupación por los riesgos de privacidad online, bien sea porque hayan recibido formación precisa, o porque hayan sufrido sus consecuencias (Ahn et al., 2012; Aldrin, 2019; Emanuel y Stanton, 2015; Gardner y Davis, 2013; Herring y Kapidzic, 2015; Livingstone, 2015; Reid y Boyer, 2013; Richardson, 2017). El problema se sitúa en lo que Barth y De Jong (2017) han denominado como la "paradoja de la privacidad", que presupone que aun conociéndose los riesgos se elige participar en lugar de protegerse. La propia cultura digital, como hemos expuesto, puede estar propiciando prácticas de auto-representación que no son seguras, lo que genera tensiones en los menores sobre lo que se considera o no apropiado publicar (Mascheroni et al., 2015) porque ante la falta de formación son los menores los que están construyendo sus propias reglas de publicación, a veces impulsivas, reaccionarias o rutinarias (Spiller, 2020). A ello hay que añadir las necesidades de su momento vital, que propician estas prácticas de auto-revelación y exhibición, porque en la adolescencia sus comportamientos suelen estar dirigidos por el deseo de atención o la evitación de la exclusión de su grupo de pertenencia (Eek-Karlsson, 2021). Ante los riesgos presentes, con independencia de si es por cultura, o por 
RLCS, Revista Latina de Comunicación Social, 79, 133-154

[Investigación] DOI: 10.4185/RLCS-2021-1528| ISSN 1138-5820| Año 2021

desarrollo madurativo, continuar la formación en competencias digitales sigue siendo ineludible para la protección de la privacidad de los menores.

\section{Objetivos}

A partir de lo expuesto, el objetivo principal de este estudio ha sido analizar los riesgos de privacidad a los que se enfrentan los adolescentes cuando realizan diferentes prácticas de auto-representación digital (compartir foto, nombre, o ubicación, filtrado de audiencias, mostrar congruencia) en las redes sociales que más usan. Concretamente, en este estudio se han planteado como objetivos específicos de análisis (1) comprobar si existen o no diferencias por género o edad respecto a la frecuencia con la que los adolescentes realizan dichas prácticas de riesgo al compartir información en las redes sociales que usan de manera intensiva (WhatsApp, Instagram y Spotify, según los adolescentes de la muestra); (2) si perciben o no la existencia de riesgos al compartir información personal; y, de manera complementaria, (3) si contar con formación en la competencia digital sobre protección de la privacidad online tiene algún impacto en los riesgos, concretamente estudiando si las prácticas de riesgo son más evidentes para aquellos que manifiestan que no recibieron formación en este ámbito.

\section{Metodología}

El estudio y los datos que se presentan forman parte de una investigación que ha empleado una metodología cuantitativa, mediante un cuestionario operacionalizado en consenso entre los investigadores del proyecto (CONECT-ID). Cada dimensión se generó a partir de conceptos y variables, utilizando escalas tipo Likert y preguntas dicotómicas, considerando los resultados de un análisis cualitativo previo del mismo proyecto (Muñoz-Rodríguez et al, 2020).

Para identificar cuestiones y preguntas susceptibles de error, previo a la codificación del instrumento se realizó una prueba piloto con 15 adolescentes a través de una muestra incidental, con el consentimiento de sus tutores legales, que modificó la redacción de algunos ítems. Así mismo, fue validado por ocho investigadores de las áreas de: sociología, psicología y métodos de investigación en educación. Finalmente, el cuestionario se redujo a 19 ítems y cinco bloques: datos sociodemográficos (apartado a, 6 ítems, con preguntas dicotómicas), trayectoria escolar (apartado b, 4 ítems, con escalas de 3 y 4 elementos), escenarios y usos de conexión (apartado c, 4 ítems, con escalas de 3 y 4 elementos), identidad digital (apartado d, 2 ítems, con preguntas dicotómicas) y consecuencias y resultados (apartado e, 3 ítems, con escalas de 3 y 4 elementos). Para medir la consistencia interna del instrumento se aplicó el estadístico Alfa de Cronbach, que indicó que la fiabilidad ofrecía garantías, con un valor de 0.713. Para este estudio se han seleccionado ítems de los bloques: aspectos demográficos (edad y género), escenarios y usos de conexión (uso de las redes sociales y plataformas), identidad digital (un ítem con cinco actividades de auto-representación online), y consecuencias del uso de las redes sociales (dos ítems: "compartir información personal en redes supone riesgo", y "me han formado para saber qué información compartir en internet y cuál no").

El cuestionario final fue aplicado entre noviembre y diciembre de 2020, mediante encuesta online garantizando el anonimato de los participantes y con el consentimiento de sus tutores legales, a partir de un muestreo estratificado y por conglomerados de distintas comunidades españolas, en centros de enseñanza secundaria (los profesores responsables de cada centro educativo participante compartieron las indicaciones y el cuestionario por correo electrónico a los estudiantes de su grupo). Una vez depurada se obtuvieron 2066 unidades válidas de 15 Comunidades Autónomas y 31 provincias. La muestra final presenta un equilibrio aceptable en cuanto variables sociodemográficas como sexo, edad, ámbito de residencia y clase social. Los 2066 adolescentes se encuentran entre el 
primer y cuarto curso $\left(1^{\text {ero }} 14,4 \%, 2^{\text {do }} 12,7 \% ; 3^{\text {ero }} 13,75 \%, 4^{\text {to }} 17,2 \%\right)$ de Educación Secundaria Obligatoria (ESO), primero $(19,6 \%)$ y segundo $(18,4 \%)$ de Bachillerato y Formación Profesional (FP) básica $(0.9 \%)$ y de grado medio $(3,1 \%)$. En la Tabla 1 se presenta la distribución de la muestra con respecto al género, con un 42,8\% de hombres y un 57,2\% mujeres, y las edades de 12 a 18 años. Para operativizar los análisis se ha procedido posteriormente a agrupar la edad en tres rangos (12-14; 15-16 y 17-18 años, respectivamente).

Tabla 1. Distribución de la muestra por edad y género

\begin{tabular}{|c|c|c|c|c|c|c|}
\hline \multicolumn{5}{|c|}{ Género } & \multicolumn{2}{|c|}{ Total } \\
\hline Edad (años) & $\begin{array}{c}\text { Varó } \\
\mathrm{n}\end{array}$ & $\%$ & $\begin{array}{c}\text { Muje } \\
\text { r }\end{array}$ & $\%$ & $\mathrm{~N}$ & $\%$ \\
\hline 12 & 143 & 49.1 & 148 & 50.9 & 291 & 14 \\
\hline 13 & 104 & 43.3 & 136 & 56.7 & 240 & 11.6 \\
\hline 14 & 133 & 47.8 & 145 & 52.2 & 278 & 13.45 \\
\hline 15 & 144 & 42.7 & 193 & 57.3 & 337 & 16.30 \\
\hline 16 & 183 & 40 & 276 & 60 & 459 & 22.21 \\
\hline 17 & 152 & 39 & 238 & 61 & 390 & 18.87 \\
\hline 18 & 25 & 35.2 & 46 & 64.8 & 71 & 3.43 \\
\hline Total & 884 & 42.8 & 1182 & 57.2 & 2066 & 100 \\
\hline \multicolumn{7}{|c|}{ Nota. N de la muestra 2066.} \\
\hline
\end{tabular}

Fuente: Elaboración propia

El procedimiento para la selección de las redes sociales que los adolescentes utilizan de forma intensiva fue el siguiente: en el apartado b) de escenarios y usos de conexión, para cada red social se pidió a los participantes que especificaran si realizaban un "uso intensivo" (frecuencia continuada de uso y para publicar videos, fotos o comentarios) "uso limitado" (menor frecuencia y para revisar información de otros) o "no uso"; los datos mostraron, como se observa en el Gráfico 1, que los adolescentes eran usuarios intensivos de tres redes sociales principalmente: WhatsApp (59,8\%), Instagram (54,4\%) y Spotify (o plataformas de música similares) (54\%), siendo el uso de otras redes más limitado. Para asegurar representatividad se realizó un análisis diferencial por género y el valor estadístico Chi-Cuadrado $\left(X^{2}\right)$ determinó que no existían diferencias significativas entre el género y el uso intensivo, uso limitado o no uso de WhatsApp $(p=0,713)$, Instagram $(p=0,901)$ y Spotify $(p=0165)$. 
RLCS, Revista Latina de Comunicación Social, 79, 133-154

[Investigación] DOI: 10.4185/RLCS-2021-1528| ISSN 1138-5820| Año 2021

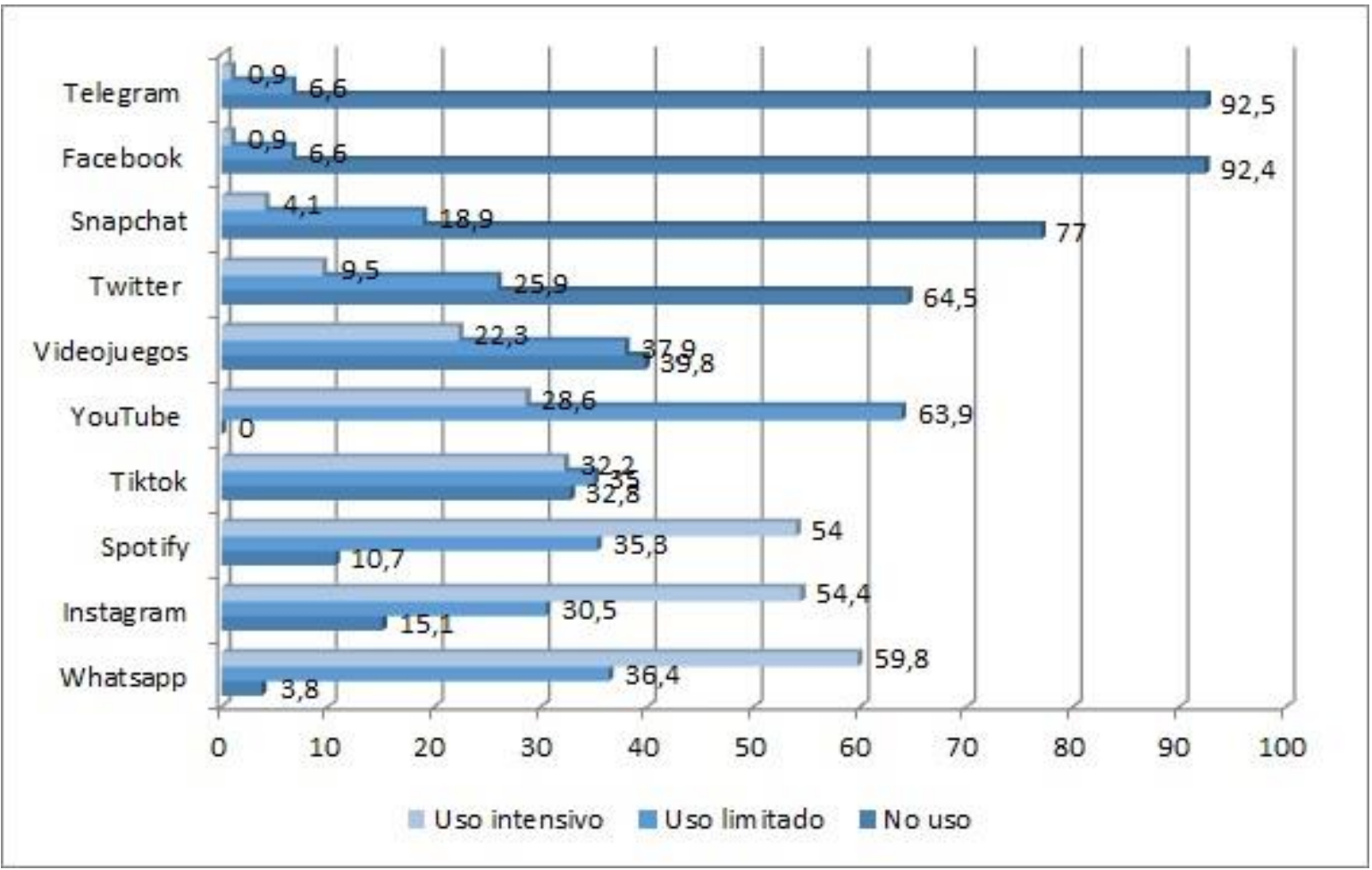

Gráfico 1: Representación del uso de diferentes redes sociales por la muestra

Fuente: Elaboración propia

Extrayendo a los adolescentes que hacen un uso más intensivo de las tres redes sociales, tanto en tiempo como en diversidad de prácticas, se han utilizado para este estudio submuestras de $\mathrm{n}=1236$ para WhatsApp, de $\mathrm{n}=1124$ para Instagram y de $\mathrm{n}=1116$ para Spotify, sobre la muestra original de 2066 sujetos. Se realizaron análisis separados en las tres redes sociales dado que cada una puede permitir prácticas de auto-representación diferentes, tales como: compartir foto, ubicación, etc.

En la tabla 2 se presenta la descripción de las tres submuestras, por edad y género, de acuerdo al primer objetivo de análisis que busca encontrar resultados diferenciales, habiéndose garantizado buena proporcionalidad para estas variables respecto a la muestra global.

Tabla 2. Redes sociales de uso intensivo y distribución por género y edad.

\begin{tabular}{|c|c|c|c|c|c|c|}
\hline \multirow{2}{*}{ Red social } & $12-14$ & $15-16$ & $17-18$ & Hombre & Mujer & Total \% \\
\cline { 2 - 7 } WhatsApp & $\begin{array}{c}39,6 \% \\
(\mathrm{n}=490)\end{array}$ & $\begin{array}{c}38,2 \% \\
(\mathrm{n}=472)\end{array}$ & $\begin{array}{c}22,2 \% \\
(\mathrm{n}=274)\end{array}$ & $\begin{array}{c}43,4 \% \\
(\mathrm{n}=536)\end{array}$ & $\begin{array}{c}56,6 \% \\
(\mathrm{n}=700)\end{array}$ & $\begin{array}{c}100 \\
(\mathrm{n}=\mathbf{1 2 3 6})\end{array}$ \\
\hline Instagram & $\begin{array}{c}39,1 \% \\
(\mathrm{n}=439)\end{array}$ & $\begin{array}{c}38,9 \% \\
(\mathrm{n}=437)\end{array}$ & $\begin{array}{c}22,1 \% \\
(\mathrm{n}=248)\end{array}$ & $\begin{array}{c}42,3 \% \\
(\mathrm{n}=476)\end{array}$ & $\begin{array}{c}57,7 \% \\
(\mathrm{n}=648)\end{array}$ & $\begin{array}{c}100 \\
(\mathbf{n}=\mathbf{1 1 2 4})\end{array}$ \\
\hline Spotify & $\begin{array}{c}38,1 \% \\
(\mathrm{n}=426)\end{array}$ & $\begin{array}{c}39,3 \% \\
(\mathrm{n}=439)\end{array}$ & $\begin{array}{c}22,5 \% \\
(\mathrm{n}=251)\end{array}$ & $\begin{array}{c}41,7 \% \\
(\mathrm{n}=463)\end{array}$ & $\begin{array}{c}58,5 \% \\
(\mathrm{n}=653)\end{array}$ & $\begin{array}{c}100 \\
(\mathrm{n}=\mathbf{1 1 1 6})\end{array}$ \\
\hline Total sobre N & $\begin{array}{c}39,2 \% \\
(\mathrm{n}=809)\end{array}$ & $\begin{array}{c}38,5 \% \\
(\mathrm{n}=796)\end{array}$ & $\begin{array}{c}22,3 \% \\
(\mathrm{n}=461)\end{array}$ & $\begin{array}{c}42,8 \% \\
(\mathrm{n}=884)\end{array}$ & $\begin{array}{c}57,2 \% \\
(\mathrm{n}=1182)\end{array}$ & \\
\hline
\end{tabular}

Fuente: Elaboración propia 
Para el segundo objetivo de análisis se ha tenido en cuenta la percepción del riesgo por parte de los adolescentes (ítem "compartir información personal en redes supone riesgo") analizando de manera diferenciada si su percepción varía en función de las tres redes sociales que utilizan de manera intensiva, así como la existencia de diferencias en dicha percepción según edad y género.

El procedimiento para el análisis del objetivo complementario sobre el efecto de la formación ha sido también mediante selección de una submuestra a partir del ítem que refiere a la competencia digital asociada a haber recibido formación sobre privacidad ("me han formado para saber qué información compartir en internet y cuál no"). El ítem, formato tipo Likert, halló que el 56,3\% estaba totalmente de acuerdo, el 25,8\% bastante de acuerdo, el 9,8\% poco de acuerdo y el 8,1\% nada de acuerdo; el perfil de estos últimos sujetos, que no habrían recibido formación, correspondientes a $n=168$, fue analizado respecto a la exposición de riesgos de auto-representación, comparativamente respecto al resto de sujetos.

En el análisis de datos se realizaron estudios descriptivos (frecuencias) y de contraste para identificar diferencias (Chi y $V$ de Cramer) por género, edad, percepción del riesgo y formación recibida. Los análisis se realizaron mediante el programa estadístico SPSS en su versión 25 , estableciéndose un nivel de significación de $\mathrm{p} \leq 0.05$.

\section{Resultados}

Para el primer objetivo se realizaron análisis diferenciados en las tres redes sociales de uso intensivo de los adolescentes de la muestra: WhatsApp, Instagram y Spotify. En primer lugar, el análisis de la frecuencia de riesgo de prácticas de auto-representación de los que usan intensivamente WhatsApp (ver, Tabla 3) mostró altos porcentajes en todos los rangos de edad con respecto al uso de fotos reales a la hora de comunicarse mediante esta red, si bien el rango con el porcentaje más alto $(41,8 \%)$ se situó en los más jóvenes ( $\leq 14$ años), siendo un $81,4 \%$ con respecto a la edad total del rango. Destacamos que el 41,2\% de los estudiantes entre 12 y 14 años indicó que prefiere no utilizar un nombre y una fotografía con las que no se les reconozca $(82,2 \%$ de $n=490$ del rango) prefiriendo entonces mostrar su foto real. Además, en cuanto a compartir su información personal, como ubicación, fotografías y aficiones, el 40.8\% (50,6\% $\mathrm{n}=490)$ de los adolescentes del mismo rango indicaron que sí, mientras que el 39.3\% del rango 15-16 años prefiere no compartirlo (52,3\%, $\mathrm{n}=472$ ). Con respecto al filtrado de las audiencias que podrían visualizar los datos personales, se distingue valores altos en la no preferencia de esta práctica de auto-representación en todos los rangos de edad, pudiendo indicar que la información visible es de dominio público y no restringida a ciertos contactos. Finalmente, se observó que todos los adolescentes buscan cierta congruencia en la auto-representación on y offline, ya que consideran que se comportan igual en la red que en persona, destacando el rango $12-14$ con un $40.3 \%$. 
RLCS, Revista Latina de Comunicación Social, 79, 133-154

[Investigación] DOI: 10.4185/RLCS-2021-1528| ISSN 1138-5820| Año 2021

Tabla 3. Prácticas de auto-representación online en WhatsApp en función de rango de edad

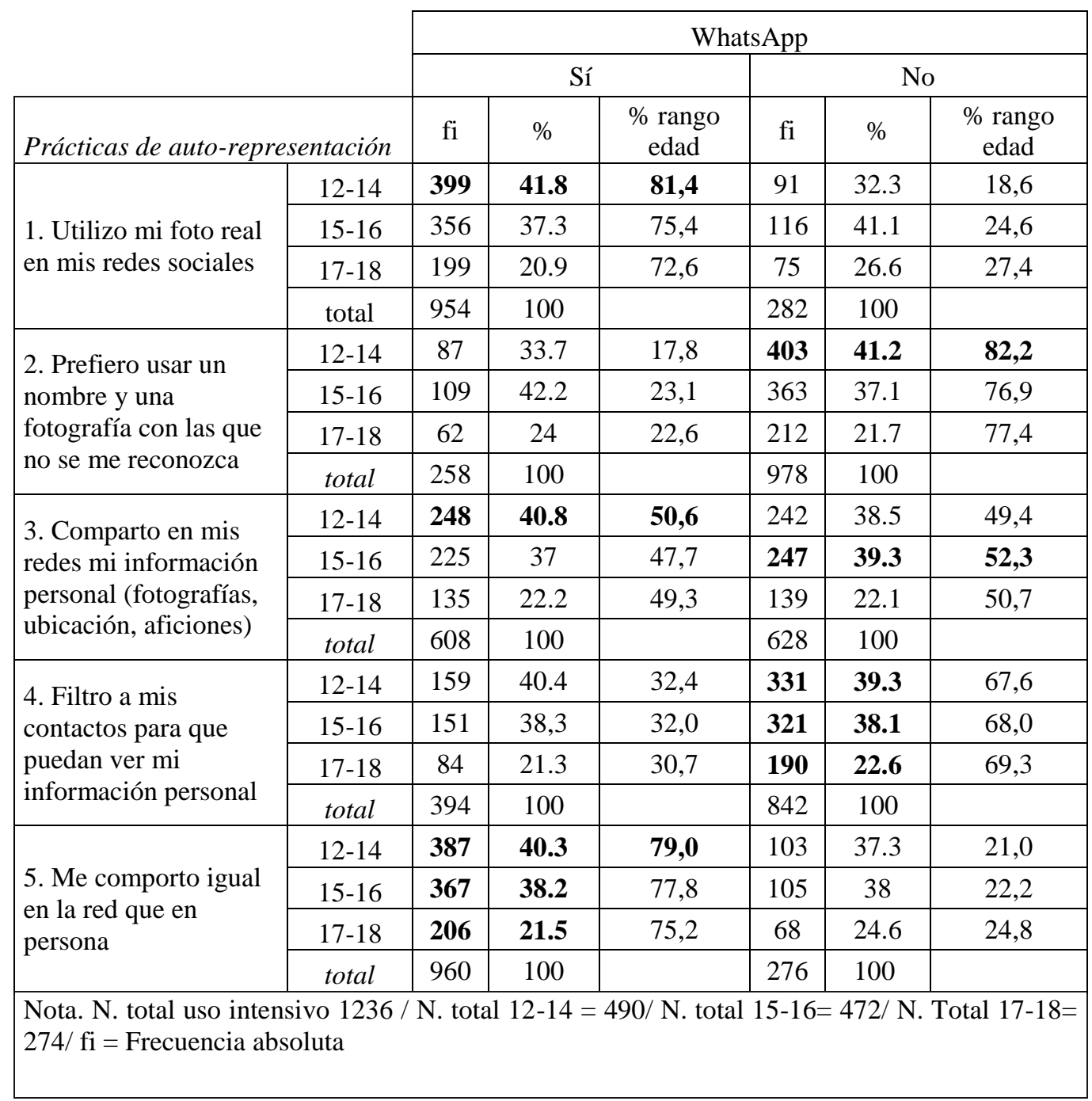

Fuente: Elaboración propia.

Con respecto a la red social Instagram (ver, Tabla 4), la mayoría de los adolescentes indicaron que utilizan sus fotos reales cuando se comunican, destacando el rango de edad 12-14 años con un $40,4 \%$, mientras que, por lo contrario, el 42,4\% de los adolescentes en el rango de edad 15-16 años no las utiliza. Se aprecia que en todas las edades prefieren utilizar sus nombres y fotografías personales para ser reconocidos, destacando ser una práctica más frecuente en los adolescentes de 12-14 años $(79,7 \%, \mathrm{n}=439)$. Confirmando lo anterior los adolescentes del grupo 12-14 años (40.2 $\%)$ y 17-18 años $(23,1 \%)$, sí comparten otros datos personales como la ubicación; en cambio los estudiantes de 15-16 años mencionan que no lo hacen. Respecto a otras prácticas de autorepresentación se continúa observando acciones de riesgos como no filtrar los contactos para que puedan visualizar los datos personales en todos los rangos de edad, con porcentajes cerca del 70\%, siendo los estudiantes de entre 15 -16 años los más representativos con un 39,1\% $(69,6 \%, n=437)$. Para finalizar se observa que los adolescentes que hacen un uso intensivo de esta red social también se comportan igual que en persona, lo que indicaría su preocupación por la congruencia, si bien los porcentajes serían más bajos que los hallados para la red social WhatsApp. 
RLCS, Revista Latina de Comunicación Social, 79, 133-154

[Investigación] DOI: 10.4185/RLCS-2021-1528| ISSN 1138-5820| Año 2021

Tabla 4. Prácticas de auto-representación online en Instagram en función de rango de edad

\begin{tabular}{|c|c|c|c|c|c|c|c|}
\hline \multirow{4}{*}{\multicolumn{2}{|c|}{ Prácticas de auto-representación }} & \multirow{2}{*}{\multicolumn{6}{|c|}{ Instagram }} \\
\hline & & & & & & & \\
\hline & & \multicolumn{3}{|c|}{ Sí } & \multicolumn{3}{|c|}{ No } \\
\hline & & fi & $\%$ & $\begin{array}{c}\% \text { rango } \\
\text { edad }\end{array}$ & fi & $\%$ & $\begin{array}{c}\% \text { rango } \\
\text { edad }\end{array}$ \\
\hline \multirow{4}{*}{$\begin{array}{l}\text { 1. Utilizo mi foto real } \\
\text { en mis redes sociales }\end{array}$} & $12-14$ & 345 & 40.4 & 78,6 & 94 & 34.9 & 21,4 \\
\hline & $15-16$ & 323 & 37.8 & 73,9 & 114 & 42.4 & 26,1 \\
\hline & $17-18$ & 187 & 21.9 & 75,4 & 61 & 22.7 & 24,6 \\
\hline & total & 855 & 100 & & 269 & 100 & \\
\hline \multirow{4}{*}{$\begin{array}{l}\text { 2. Prefiero usar un } \\
\text { nombre y una } \\
\text { fotografía con las que } \\
\text { no se me reconozca }\end{array}$} & $12-14$ & 89 & 35.7 & 20,3 & 350 & 40 & 79,7 \\
\hline & $15-16$ & 107 & 43 & 24,5 & 330 & 37.7 & 75,5 \\
\hline & $17-18$ & 53 & 21.3 & 21,4 & 195 & 22.3 & 78,6 \\
\hline & total & 249 & 100 & & 875 & 100 & \\
\hline \multirow{4}{*}{$\begin{array}{l}\text { 3. Comparto en mis } \\
\text { redes mi información } \\
\text { personal (fotografías, } \\
\text { ubicación, aficiones) }\end{array}$} & $12-14$ & 214 & 40.2 & 48,7 & 225 & 38 & 51,3 \\
\hline & $15-16$ & 195 & 36.7 & 44,6 & 242 & 40.9 & 55,4 \\
\hline & $17-18$ & 123 & 23.1 & 49,6 & 125 & 21.1 & 50,4 \\
\hline & total & 532 & 100 & & 592 & 100 & \\
\hline \multirow{4}{*}{$\begin{array}{llr}\text { 4. Filtro } & \text { a } & \text { mis } \\
\text { contactos para } & \text { que } \\
\text { puedan ver } & \text { mi } \\
\text { información personal }\end{array}$} & $12-14$ & 138 & 39.9 & 31,4 & 301 & 38.7 & 68,6 \\
\hline & $15-16$ & 133 & 38.4 & 30,4 & 304 & 39.1 & 69,6 \\
\hline & $17-18$ & 75 & 21.7 & 30,2 & 173 & 22.2 & 69,8 \\
\hline & total & 346 & 100 & & 778 & 100 & \\
\hline \multirow{4}{*}{$\begin{array}{l}\text { 5. Me comporto igual } \\
\text { en la red que en } \\
\text { persona }\end{array}$} & $12-14$ & 339 & 39.4 & 77,2 & 100 & 38 & 22,8 \\
\hline & $15-16$ & 330 & 38.3 & 75,5 & 107 & 4.7 & 24,5 \\
\hline & $17-18$ & 192 & 22.3 & 77,4 & 56 & 21.3 & 22,6 \\
\hline & total & 861 & 100 & & 263 & 100 & \\
\hline
\end{tabular}

Fuente: Elaboración propia.

En el caso de la multiplataforma Spotify -que es considera por los adolescentes como red social, ya que además de ofrecer acceso a canciones y contenidos musicales, es utilizada para compartir listas de canciones a través de un perfil público que se puede retroalimentar mediante valoraciones- la Tabla 5 presenta valores altos en los más jóvenes (12-14 años) en las prácticas uno y dos de uso de fotos reales y uso de nombre reconocido. También destacan ligeras diferencias entre los rangos de edad con respecto a compartir información personal; el 39,2\% (50,2\%, $n=246)$ de los estudiantes entre 12-14 años son los que más realizan esta acción, frente al grupo de 17-18 años $(23,5 \%)$ que no lo practican. Con respecto a los filtros de contactos y el comportamiento congruente, se mantiene la tendencia con respecto a otras redes sociales, obteniéndose que los menores no filtran sus audiencias (siendo más frecuente en el rango 12-14 años con 38.3\%) y se comportan de igual manera que en la vida real. 
RLCS, Revista Latina de Comunicación Social, 79, 133-154

[Investigación] DOI: 10.4185/RLCS-2021-1528| ISSN 1138-5820| Año 2021

Tabla 5. Prácticas de auto-representación online en Spotify en función de rango de edad

\begin{tabular}{|c|c|c|c|c|c|c|c|}
\hline & & & & & & & \\
\hline \multirow[b]{3}{*}{$\begin{array}{l}\text { Prácticas de } \\
\text { representación }\end{array}$} & \multirow[b]{3}{*}{ auto- } & \multicolumn{6}{|c|}{ Spotify } \\
\hline & & \multicolumn{3}{|c|}{ Sí } & \multicolumn{3}{|c|}{ No } \\
\hline & & fi & $\%$ & $\begin{array}{c}\% \text { rango } \\
\text { edad }\end{array}$ & fi & $\%$ & $\begin{array}{c}\text { \% rango } \\
\text { edad }\end{array}$ \\
\hline \multirow{4}{*}{$\begin{array}{l}\text { 1. Utilizo mi foto real } \\
\text { en mis redes sociales }\end{array}$} & $12-14$ & 343 & 40.2 & 80,5 & 83 & 31.6 & 19,5 \\
\hline & $15-16$ & 325 & 38.1 & 74,0 & 114 & 43.3 & 26,0 \\
\hline & $17-18$ & 185 & 21.7 & 73,7 & 66 & 25.1 & 26,3 \\
\hline & total & 853 & 100 & & 263 & 100 & \\
\hline \multirow{4}{*}{$\begin{array}{l}\text { 2. Prefiero usar un } \\
\text { nombre y una } \\
\text { fotografía con las que } \\
\text { no se me reconozca }\end{array}$} & $12-14$ & 81 & 34.2 & 19,0 & 345 & 39.2 & 81,0 \\
\hline & $15-16$ & 102 & 43 & 23,2 & 337 & 38.3 & 76,8 \\
\hline & $17-18$ & 54 & 22.8 & 21,5 & 197 & 22.4 & $\mathbf{7 8 , 5}$ \\
\hline & total & 237 & 100 & & 879 & 100 & \\
\hline \multirow{4}{*}{$\begin{array}{l}\text { 3. Comparto en mis } \\
\text { redes mi información } \\
\text { personal (fotografías, } \\
\text { ubicación, aficiones) }\end{array}$} & $12-14$ & 214 & 39.2 & 50,2 & 212 & 37.2 & 49,8 \\
\hline & $15-16$ & 215 & 39.4 & 49,0 & 224 & 39.3 & 51,0 \\
\hline & $17-18$ & 117 & 21.4 & 46,6 & 134 & 23.5 & 53,4 \\
\hline & total & 546 & 100 & & 570 & 100 & \\
\hline \multirow{4}{*}{$\begin{array}{l}\text { 4. Filtro a mis } \\
\text { contactos para que } \\
\text { puedan ver mi } \\
\text { información personal }\end{array}$} & $12-14$ & 135 & 37.8 & 31,7 & 291 & 38.3 & 68,3 \\
\hline & $15-16$ & 152 & 42.6 & 34,6 & 287 & 37.8 & 65,4 \\
\hline & $17-18$ & 70 & 19.6 & 27,9 & 181 & 23.8 & 72,1 \\
\hline & total & 357 & 100 & & 759 & 100 & \\
\hline \multirow{4}{*}{$\begin{array}{l}\text { 5. Me comporto igual } \\
\text { en la red que en } \\
\text { persona }\end{array}$} & $12-14$ & 330 & 38.8 & 77,5 & 96 & 36.2 & 22,5 \\
\hline & $15-16$ & 328 & 38.5 & 74,7 & 111 & 41.9 & 25,3 \\
\hline & $17-18$ & 193 & 22.7 & 76,9 & 58 & 21.9 & 23,1 \\
\hline & total & 851 & 100 & & 265 & 100 & \\
\hline
\end{tabular}

Fuente: Elaboración propia.

En cuanto al género, no se hallaron diferencias estadísticamente significativas, entendiendo que tanto hombres como mujeres realizan similares prácticas de auto-representación en las diferentes redes sociales. Como se observa en el gráfico 2, los y las adolescentes realizan en similares porcentajes prácticas que les colocan ante diferentes riesgos de privacidad, siendo las más frecuentes compartir sus fotos $(76,1 \%$ mujeres y $77,8 \%$ hombres) y su nombre real (79,5\% mujeres y 78,2\% hombres). 
RLCS, Revista Latina de Comunicación Social, 79, 133-154

[Investigación] DOI: 10.4185/RLCS-2021-1528| ISSN 1138-5820| Año 2021

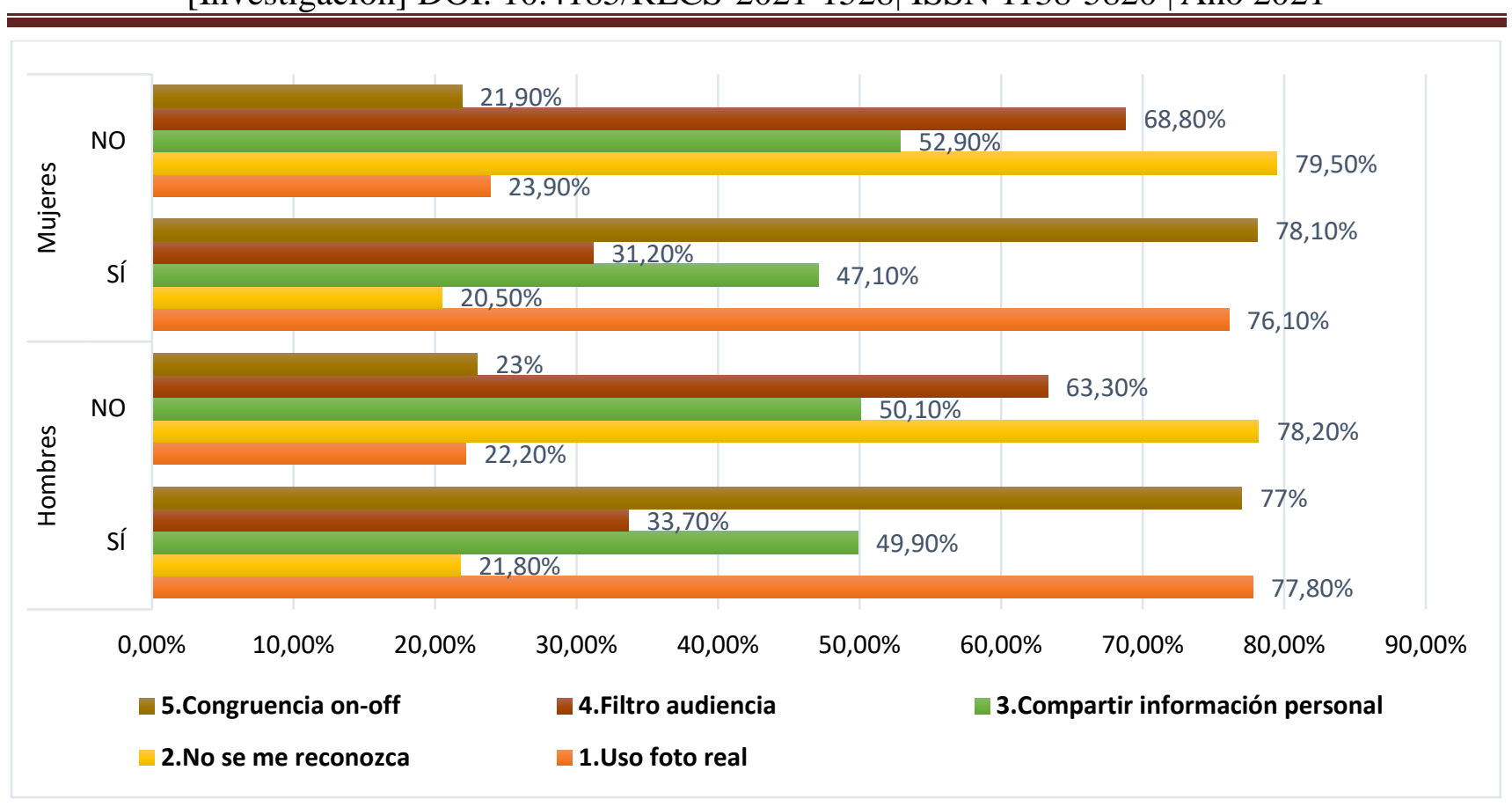

Gráfico 2: Prácticas de auto-representación online por género

Fuente: Elaboración propia

Respecto al segundo objetivo de análisis sobre la percepción del riesgo que los adolescentes tienen sobre la privacidad ("compartir mi información personal con mis amigos a través de internet supone riesgo"), de manera diferenciada en las tres redes sociales, se observaron porcentajes similares en el grado de acuerdo (ver, Tabla 6). No existieron diferencias estadísticamente significativas por edad, ni por género. Los porcentajes no difieren respecto a la muestra total, hallándose un consenso de entorno al $70 \%$ de adolescentes que reconocerían que al compartir información personal en redes sociales se exponen a riesgos, siendo más elevado el porcentaje de acuerdo en las respuestas halladas para los hombres usuarios intensivos de Instagram. No obstante, el porcentaje de desacuerdo en torno al 30\%, tanto en hombres como en mujeres, usuarios de las tres redes, resulta un dato relevante respecto a la existencia de adolescentes que aún mantienen una baja conciencia ante los riesgos.

Tabla 6. Percepción de riesgo al compartir información personal en redes sociales

\begin{tabular}{|l|c|c|c|c|c|}
\hline & \multicolumn{2}{|c|}{ Desacuerdo } & \multicolumn{2}{c|}{ Acuerdo } & \\
\hline & Hombre & Mujer & Hombre & Mujer & $X^{2}$ \\
\hline WhatsApp $(\mathrm{n}=1236)$ & $151(28,17 \%)$ & $219(31,28 \%)$ & $385(71,82 \%)$ & $481(68,71 \%)$ & 0,157 \\
\hline Instagram $(\mathrm{n}=1124)$ & $130(27,31 \%)$ & $\mathbf{1 9 6}(\mathbf{3 0 , 2 4 \%})$ & $\mathbf{3 4 6}(\mathbf{7 2 , 6 8 \%})$ & $452(69,75 \%)$ & 0,398 \\
\hline Spotify $(\mathrm{n}=1116)$ & $139(30,02 \%)$ & $193(29,55 \%)$ & $324(69,97 \%)$ & $460(70,44 \%)$ & 0,968 \\
\hline Total $(\mathrm{N}=2066)$ & $264(29,86)$ & $355(30,03 \%)$ & $620(70,13 \%)$ & $827(69,96 \%)$ & 0,884 \\
\hline
\end{tabular}

Fuente: Elaboración propia

Finalmente, para el análisis del efecto protector de la formación recibida sobre la privacidad online, se comprobó que no existían diferencias entre las distintas redes sociales y se realizó un análisis en profundidad de los adolescentes que manifestaron no haber recibido formación ( $\mathrm{n}=168$, correspondiente al $8,1 \%$ de la muestra total). En cuando a la edad, el 34,5\% correspondía al rango 
12-14 años, el 45,8\% al rango 15-16 años y el 19,6\% al rango 17-18 años. Por género no se hallaron diferencias, el 44,6\% de hombres y el 55,4\% de mujeres indicó no haber recibido formación. Respecto a las prácticas de auto-representación, se obtuvieron porcentajes ligeramente superiores que los que se ha presentado para las diferentes redes sociales: el 76,8\% comparten su foto, el $78 \%$ usan su nombre real, el $41,1 \%$ comparten su ubicación, el $64,3 \%$ no filtra la audiencia, y el $84,5 \%$ se preocupa por la impresión de congruencia on-offline, todo lo cual estaría indicando que la falta de formación les situaría en una posición más vulnerable respecto a la protección de su privacidad online. Sin embargo, estas diferencias no resultaron significativas a nivel estadístico, por lo que el efecto preventivo o protector de la formación no resultaría confirmado.

\section{Discusión/Conclusiones}

Los resultados de este estudio ponen de manifiesto que los adolescentes están realizando prácticas de auto-representación digital que les exponen ante ciertos riesgos, puesto que en ellas están publicando información personal que puede comprometer su privacidad, especialmente, entre las más frecuentes está el uso de fotos, seguido de la necesidad de congruencia, como también han señalado otros estudios (Aldrin, 2019; Doster, 2013; Hong et al., 2020). En cuanto a lo último, es importante señalar que cada vez más estudios confirman que las redes sociales ya no son espacios de experimentación de una identidad diferente, sino una continuación on-offline, desechándose los comportamientos irreales o exagerados por otros que son más congruentes (Leong, 2016). En la misma línea, se hallaron porcentajes elevados en torno al $80 \%$ en cuando al hecho de preferir usar nombres o fotos reales, lo que indicaría que los adolescentes ya no pretenden esconderse en identidades falsas, como confirmaban otras investigaciones (Bell, 2019; Crescenzi et al., 2013). Hay otras prácticas de representación que no son tan frecuentes, pero cuyos porcentajes son más elevados entre el colectivo de adolescentes más joven (de 12 a 14 años), referidas al hecho de compartir su localización y sus fotos, o aficiones, que sería una actividad que la mitad de ellos practican con frecuencia. Hay que tener en cuenta que este porcentaje puede estar condicionado por el momento de recogida de los datos, en el contexto Covid-19, posconfinamiento, donde una de las prácticas frecuentes para los menores ha sido compartir retos (challenges) en redes sociales (Yang et al., 2018). Se debe seguir investigando este resultado en un momento posterior y verificar si ha sido una tendencia contextualizada en ese momento, o se trata de una práctica frecuente y/o creciente entre los menores.

Otra de las cuestiones relevantes en cuanto a sus prácticas de auto-representación, que además ha sido suficientemente analizada en estudios con colectivos similares (Baym y Boyd, 2012; Richardson, 2017; Rodríguez et al., 2017; Wood et al., 2016) es el control que ofrece al usuario de las redes sociales la posibilidad de limitar la publicación de información personal para diferentes audiencias, una acción que no ha resultado ser una práctica común en los adolescentes de la muestra analizada, ya que en torno al $70 \%$ confirmaron que no la realizan. Esto podría suponer que, efectivamente, sus prácticas suponen riesgos para su privacidad, porque su información personal es de acceso público y no limitada a sus contactos. Otra explicación posible, como hemos comentado anteriormente, es que los adolescentes puedan estar más preocupados por la coherencia y la continuidad de su mismo yo "on-offline" sin necesidad de ocultar información o generar incongruencias. En cualquier caso, y aunque no se hayan encontrado diferencias estadísticamente significativas, los porcentajes aumentan con la edad, es decir, que cuanto más mayores menos filtran los contactos, de lo que se podría advertir que comienzan exponiendo menos su información personal y con el tiempo van perdiendo esta conducta protectora.

A pesar de haber realizado el análisis diferenciado en función de las tres redes que usan con mayor frecuencia, o de modo intensivo (WhatsApp, Instagram, Spotify), los porcentajes hallados y la no existencia de diferencias estadísticamente significativas nos llevarían a afirmar que no son las 
posibilidades tecnológicas las que les exponen ante los riesgos de privacidad, ya que aunque las redes ofrezcan diferentes opciones de auto-representación en todas se comportan de manera similar. Una explicación posible puede relacionarse con las necesidades y expectativas evolutivas propias de la adolescencia, que como han puesto de manifiesto otros estudios, llevarían a los adolescentes a comportarse de manera similar como resultado de la pertenencia a cultura de la exhibición y la comparación social (Mascheroni et al., 2015; Spiller, 2020; Yang et al., 2018).

En cuanto a la edad, es importante indicar que buena parte de los porcentajes que suponen frecuencias elevadas en prácticas de auto-representación digital asociadas a riesgos se sitúan en el rango de 12-14 años, para las tres redes sociales analizadas. No se obtuvieron correlaciones que avalen que cuanto más jóvenes son, más expuestos a los riesgos estarían en sus prácticas, pero el hecho de que sean ligeramente más frecuentes invita a reflexionar sobre la necesidad de desarrollar formación preventiva y protectora cuanto antes, incluso en etapas de educación primaria o elemental.

Respecto al género, si bien otros estudios confirmaron que las mujeres suelen mostrar conductas más protectoras (Herring y Kapidzic, 2015; Rodríguez et al., 2017), en la muestra no se hallaron diferencias estadísticamente significativas, precisándose seguir realizando estudios que confirmen o no los resultados de esta investigación.

De cara a la necesidad de desarrollar competencias digitales que hagan más conscientes a los adolescentes de los riesgos online, nuestros resultados confirman la paradoja de privacidad expuesta por Barth y De Jong (2017), ya que los participantes sí manifiestan en su gran mayoría que son conocedores de la existencia de riesgos cuando comparten su información personal, sin que este conocimiento les prevenga de desarrollar acciones que serían arriesgadas. Es probable que, por desarrollo evolutivo, o por presión social y cultural (Eek-Karlsson, 2021), puedan acabar sacrificando su protección por la necesidad de obtener prestigio social o popularidad; por ello, las acciones educativas deberían trabajar estos aspectos sobre los riesgos, desarrollando la necesidad de valorar la protección también como una necesidad. En esta línea, algunas iniciativas existentes (Gairín y Mercader, 2017) se dirigen a prevenir o detectar las conductas de riesgos en el uso de contenidos digitales y tecnológicos entre adolescentes, potenciando la autoprotección (aumento de medidas de privacidad y protección de datos personales en las redes sociales), las restricciones (favorecer control parental y gestión del tipo de páginas de acceso que hacen los menores); el uso de antivirus (promover el uso adecuado de antivirus para evitar que se robe la identidad digital a través de perfiles); la creación de reglas y normas como son no compartir información o fotografía a desconocidos; y el establecimiento de horarios pactados para la conexión lúdica y la promoción de zonas comunes en el hogar que controles el uso excesivo o abusivo de las tecnologías.

La formación en conductas protectoras, integrada en las competencias digitales básicas, ayudaría a los menores a saber cómo interactuar de forma segura en las redes sociales, con responsabilidad y cuidando su privacidad y auto-representación digital. Sin embargo, es sólo un factor protector potencial, debiéndose analizar y reforzar el tipo de formación que reciben los menores; en nuestro estudio, contrariamente a lo esperado, el análisis de aquellos adolescentes que manifestaron no haber recibido formación para protegerse de riesgos de privacidad online no confirmó un efecto diferencial, que denotara la existencia de mayores prácticas de riesgo, respecto a otros que sí declararon haber recibido formación, se encontraron porcentajes similares. Este resultado debe ser rebatido en nuevos estudios, ya que también hay investigaciones que han afirmado lo contrario, exponiendo que empieza a ser evidente en los adolescentes y jóvenes el desarrollo de conductas protectoras ante la "vigilancia imaginada" (Adorjan y Ricciardelli, 2020; Duffy y Chan, 2019), como resultado de la concientización ante los riesgos del Bigdata, que también se incluyen los asociados a la protección 
de la privacidad individual, aspectos todos emergentes que tendrán claras repercusiones en las agendas educativas a corto plazo.

Para finalizar, es importante señalar que este estudio refuerza la necesidad de seguir desarrollando competencias digitales en los menores -en general y en particular sobre la privacidad-, en aras a limitar prácticas impulsivas o arriesgadas. Como se ha evidenciado, el uso de las redes sociales está en aumento, lo que requiere de nuevas estrategias pedagógicas que permitan a los adolescentes manejar información de forma crítica, así como diseñar y publicar contenidos compartidos de forma responsable. Para ello, es esencial enseñarles qué prácticas de auto-representación digital les harían más vulnerables ante ataques contra su privacidad, desechando las reglas construidas por ellos mismos (Spiller, 2020) por estrategias protectoras que se integren en el conjunto de competencias digitales básicas y necesarias, muchas de las cuales han sido acordadas como habilidades clave a nivel internacional. En este sentido, tan importante es la formación de los estudiantes en competencias digitales como lo es la de los profesores encargados de formarles, garantizando la calidad y eficiencia en la adquisición de dichas competencias (Padilla et al., 2020).

\section{Referencias}

Adorjan, M. y Ricciardelli, R. (2020). Cyber-Risk and Youth: Digital Citizenship, Privacy and Surveillance [Libro electrónico]. Routledge. https://www.routledge.com/Cyber-risk-and-YouthDigital-Citizenship-Privacy-and-Surveillance/Adorjan-Ricciardelli/p/book/9780367663209

Agenda 2030. (2020). Objetivos de desarrollo sostenibles (ODS). https://bit.ly/3aWPRzY

Aguaded, J. (2007). Observatics. La implementación del software libre en centros TIC andaluces. Proyecto de investigación. Universidad de Huelva, Grupo Ágora.

Ahn, J., Subramaniam, M., Fleischmann, K., Waugh, A., Walsh, G. y Druin, A. (2012). Youth identities as remixers in an online community of storytellers: Attitudes, strategies, and values. Proceedings of the American Society for Information Science and Technology, 49(1), 1-10. https://doi.org/10.1002/meet.14504901089

Aldrin, E. (2019). Naming, Identity, and Social Positioning in Teenagers' Everyday Mobile Phone Interaction. Names, 67(1), 30-39. https://doi.org/10.1080/00277738.2017.1415523

Atchoarena, D., Selwyn, N., Chakroun, B., Miaho, F., West, M. y Coligny, C. de (2017). Working Group on Education: Digital skills for life and work. UNESCO. https://broadbandcommission.org/publication/digital-skills-for-life-and-work/

Barth, S. y De Jong, M. D. (2017). The privacy paradox-Investigating discrepancies between expressed privacy concerns and actual online behavior-A systematic literature review. Telematics and informatics, 34(7), 1038-1058. https://doi.org/10.1016/j.tele.2017.04.013

Baym, N. K. y Boyd, D. (2012). Socially Mediated Publicness: An Introduction. Journal of $\begin{array}{llll}\text { Broadcasting } \text { and } \quad \text { Electronic } & \text { 320-329. }\end{array}$ https://doi.org/10.1080/08838151.2012.705200

Bell, B. T. (2019). You take fifty photos, delete forty-nine and use one: A qualitative study of adolescent image-sharing practices on social media. International Journal of Child-Computer Interaction, 20, 64-71. https://doi.org/10.1016/j.ijcci.2019.03.002 
Cabero, J., Martínez, S., Valencia, R., Leiva, J., Orellana, M. y Harvey, I. (2020). La adicción de los estudiantes a las redes sociales on-line: un estudio en el contexto latinoamericano. Revista Complutense De Educación, 31(1), 1-12. https://doi.org/10.5209/rced.61722

Casillas-Martín, S., Cabezas-González, M. y García-Valcárcel, A. (2020). Análisis psicométrico de una prueba para evaluar la competencia digital de estudiantes de Educación Obligatoria. RELIEVE, 26(2), http://doi.org/10.7203/relieve.26.2.17611

Cipolletta, S., Malighetti, C., Cenedese, C. y Spoto, A. (2020). How Can Adolescents Benefit from the Use of Social Networks? The iGeneration on Instagram. International Journal of Environmental Research and Public Health, 17(19), 6952. https://doi.org/10.3390/ijerph17196952

Crescenzi, L., Araüna, N. y Tortajada, I. (2013). Privacy, self-disclosure and self-image of Spanish teenagers on social networking sites. The case of Fotolog. Revista Comunicación y Sociedad, 26(2), 65-78. https://revistas.unav.edu/index.php/communication-and-society/article/view/36123

Davis, K. (2013). Young people's digital lives: The impact of interpersonal relationships and digital media use on adolescents' sense of identity. Computers in Human Behavior, 29(6), 2281-2293. https://doi.org/10.1016/j.chb.2013.05.022

De la Villa Moral-Jiménez, M. y Fernández-Domínguez, S. (2019). Uso problemático de Internet en adolescentes españoles y su relación con autoestima e impulsividad. Avances en Psicología Latinoamericana, 37(1), 103-119. https://dx.doi.org/10.12804/revistas.urosario.edu.co/ap1/a.5029

DeCew, J. W. (1997). In pursuit of privacy. Law, Ethics, and the Rise of Technology. Cornell University Press.

Ditchfield, H. (2020). Behind the screen of Facebook: Identity construction in the rehearsal stage of online interaction. New Media \& Society, 22(6), 927-943. https://doi.org/10.1177/1461444819873644

Doster, L. (2013). Millennial teens design and redesign themselves in online social networks. Journal of Consumer Behaviour, 12(4), 267-279. https://doi.org/10.1002/cb.1407

Duffy, B. E. y Chan, N. K. (2019). You never really know who's looking: Imagined surveillance across social media platforms. New Media \& Society, 21(1), 119-138. https://doi.org/10.1177/1461444818791318

Eek-Karlsson, L. (2021). The Importance of Belonging: A Study About Positioning Processes in Youths' Online Communication. SAGE Open, 11(1). https://doi.org/10.1177/2158244020988860

Emanuel, L. y Stanton Fraser, D. (2014). Exploring physical and digital identity with a teenage cohort. In Proceedings of the 2014 conference on interaction design and children (pp. 67-76). https://doi.org/10.1145/2593968.2593984

Ferrari, A. (2013). DIGCOMP: A Framework for Developing and Understanding Digital Competence in Europe. Sevilla: Joint Research Centre, Institute for Prospective Technological Studies. http://dx.doi.org/10.2788/52966 
RLCS, Revista Latina de Comunicación Social, 79, 133-154

[Investigación] DOI: 10.4185/RLCS-2021-1528| ISSN 1138-5820| Año 2021

Ferrés, J. y Piscitelli, A. (2012). Media Competence. Articulated Proposal of Dimensions and Indicators. Comunicar, 19(38), 75-82. https://doi.org/10.3916/C38-2012-02-08

Gairín Sallán, J. y Mercader, C. (2017). Usos y abusos de las TIC en los adolescentes. Revista de Investigación Educativa, 36(1), 125-140. https://doi.org/10.6018/rie.36.1.284001

García, M.C., Seco, J.A. y Hoyo, M. (2013). La participación de los jóvenes en las redes sociales: finalidad, oportunidades y gratificaciones. Anàlisi Monogràfic, 48,95-110. https://doi.org/10.7238/a.v0iM.1968

Gardner, H. y Davis, K. (2013). Whither digital natives. American Scholar, 82(4), 14-18.

Gioia, F., McLean, S., Griffiths, M. D. y Boursier, V. (2021). Adolescents' selfie-taking and selfieediting: A revision of the photo manipulation scale and a moderated mediation model. Current Psychology, 1-17. https://doi.org/10.1007/s12144-021-01702-x

González Calatayud, V., Román García, M. y Prendes Espinosa, M. P. (2018). Formación en competencias digitales para estudiantes universitarios basada en el modelo DigComp. Edutec. Revista Electrónica De Tecnología Educativa, (65), 1-15 (391). https://doi.org/10.21556/edutec.2018.65.1119

Herring, S. C. y Kapidzic, S. (2015). Teens, gender, and self-presentation in social media. International encyclopedia of social and behavioral sciences, 2, 1-16. https://doi.org/10.1016/B978-0-08-097086-8.64108-9

Hong, S., Jahng, M. R., Lee, N. y Wise, K. R. (2020). Do you filter who you are? Excessive selfpresentation, social cues, and user evaluations of Instagram selfies. Computers in Human Behavior, 104. https://doi.org/10.1016/j.chb.2019.106159

Jeong, Y. y Coyle, E. (2014). What are you worrying about on Facebook and Twitter? An empirical investigation of young social network site users' privacy perceptions and behaviors. Journal of Interactive Advertising, 14(2), 51-59. https://doi.org/10.1080/15252019.2014.930678

Kemp, S. (27 enero de 2021). Digital 2021. Global Overview Report. DATAREPORTAL. https://datareportal.com/reports/digital-2021-global-overview-report

Leong, L. (2016). Mobile identities: managing self and stigma in iPhone app use. Observatorio $\left(O B S^{*}\right)$, 10(1). http://obs.obercom.pt/index.php/obs/article/view/897/780

Livingstone, S., Haddon, L., Görzig, A. y Ólfsson, K. (2010). Risks and safety on the Internet: The perspective of European children. Initial Findings. EU Kids On-line. http://eprints.1se.ac.uk/33731/1/Risks\%20and\%20safety\%20on\%20the\%20internet\%281sero\%29. pdf

Livingstone, S., Mascheroni G. y E. Staksrud (2015). Developing a framework for researching children's online risks and opportunities in Europe. EU Kids Online. http://eprints.lse.ac.uk/64470/

Mascheroni, G., Vincent, J. y Jimenez, E. (2015). "Girls are addicted to likes so they post seminaked selfies": Peer mediation, normativity and the construction of identity online. 
RLCS, Revista Latina de Comunicación Social, 79, 133-154

[Investigación] DOI: 10.4185/RLCS-2021-1528| ISSN 1138-5820| Año 2021

Cyberpsychology: Journal of Psychosocial Research on Cyberspace, 9(1), 5. https://doi.org/10.5817/CP2015-1-5

McDougall, J., Zezulkova, M., Van Driel, B. y Sternadel, D. (2018). Teaching media literacy in Europe: evidence of effective school practices in primary and secondary education, NESET II report. Project Report. Publications Office of the European Union. https://nesetweb.eu/en/resources/library/teaching-media-literacy-in-europe-evidence-of-effectiveschool-practices-in-primary-and-secondary-education/

Montiel, I., Carbonell, E. y Pereda, N. (2016). Multiple online victimization of Spanish adolescents: Results from a community sample. Child Abuse \& Neglect, 52, 123-134. https://doi.org/10.1016/j.chiabu.2015.12.005

Muñoz-Rodríguez, J. M., Torrijos Fincias, P., Serrate González, S., y Murciano Hueso, A. (2020). Entornos digitales, conectividad y educación. Percepción y gestión del tiempo en la construcción de la identidad digital de la juventud. Revista Española de Pedagogía, 78(277), 457-476. https://doi.org/10.22550/REP78-3-2020-07

Núñez-Rola, C. y Ruta-Canayong, N.J. (2019). Social media influences teenagers. International journal of research science \& management, 6(6), 38-48. http://doi.org/10.5281/zenodo.3260717

Padilla, A. L.; Gámiz, V. M. ${ }^{a}$ y Romero, M. ${ }^{a}$ A. (2020). Evolución de la competencia digital docente del profesorado universitario: incidentes críticos a partir de relatos de vida. Educar, 56(1), 109-27. https://doi.org/10.5565/rev/educar.1088

Peñalva, A., Napal, M. y Mendioroz, A.M. (2018). Competencia y alfabetización digitales de los adultos (profesorado y familias). International Journal of New Education, 1(1), 1-13. https://doi.org/10.24310/IJNE1.1.2018.4892

Pérez, A. y Delgado, A. (2012). From digital and audiovisual competence to media competence: Dimensions and indicators. [De la competencia digital y audiovisual a la competencia mediática: dimensiones e indicadores]. Comunicar, 39, 25-34. https://doi.org/10.3916/C39-2012-02-02

Pontes, H. M., Szabo, A. y Griffiths, M. D. (2015). The impact of Internet based specific activities on the perceptions of Internet addiction, quality of life, and excessive usage: A cross-sectional study. Addictive Behaviors Reports, 1, 19-25. https://doi.org/10.1016/j.abrep.2015.03.002

Quintas, N. y González, A. (2021). Evolución de la medición digital de la audiencia en el mercado español: estado de la cuestión y retos de futuro. Profesional De La Información, 30(1). https://doi.org/10.3145/epi.2021.ene.02

Redecker, C. (2017). European Framework for the Digital Competence of Educators: DigCompEdu. Joint Research Centre. https://publications.jrc.ec.europa.eu/repository/handle/JRC107466

Reid, G. y Boyer, W. (2013). Social network sites and young adolescent identity development. Childhood Education, 89(4), 243-253. https://doi.org/10.1080/00094056.2013.815554

Reisenzein, R., Meyer, W. y Niepel, M. (2012). Self-disclosure. Encyclopedia of human behavior. Science-direct. $\quad$ https://www.sciencedirect.com/topics/biochemistry-genetics-and-molecularbiology/self-disclosure 
Richardson, J. M. (2017). The promposal: youth expressions of identity and 'love'in the digital age. Learning, Media and Technology, 42(1), 74-86. https://doi.org/10.1080/17439884.2016.1130055

Rodríguez, E., Pequeño, G. y Requies, I. R. (2017). Socialization of Interconnected Youth: Experiencing Identity in an Augmented Society. Profesorado revista de curriculum y formación de profesorado, 21(2), 171-190. https://core.ac.uk/display/161542137

Schwartz, R. y Halegoua, G. R. (2015). The spatial self: Location-based identity performance on $\begin{array}{lllll}\text { social media. New media \& } & \text { society, 177(10), }\end{array}$ https://doi.org/10.1177/1461444814531364

Scott, G. G. y Fullwood, C. (2020). Does recent research evidence support the hyperpersonal model of online impression management? Current Opinion in Psychology, 36, 106-111. https://doi.org/10.1016/j.copsyc.2020.05.005

Seo, H., Houston, J. B., Knight, L. A. T., Kennedy, E. J. y Inglish, A. B. (2014). Teens' social media use and collective action. New Media \& Society, 16(6), 883-902. https://doi.org/10.1177/1461444813495162

Siddiqui, S. y Singh, T. (2016). Social Media and its Impact with Positive and Negative Aspects. International Journal of Computer Applications Technology and Research, 5(2), 71-75. DOI: 10.7753/IJCATR0502.1006

Spiller, K. (2020). Putting Everything up There: Framing How We Navigate the Intricacies of Privacy and Security on Social Media. Humanity \& Society, 45(1), 3-23. https://doi.org/10.1177/0160597620904502

Statista (2021). Porcentaje de niños de entre 4 y 15 años usuarios en redes sociales en España. https://es.statista.com/estadisticas/1127004/poblacion-infantil-porcentaje-de-usuarios-en-redessociales-en-espana/

Stuart, A., Bandara, A. K. y Levine, M. (2019). The psychology of privacy in the digital age. Social and Personality Psychology Compass, 13(11). https://doi.org/10.1111/spc3.12507

Tejada, J. y Pozos, K.V. (2018). Nuevos escenarios y competencias digitales docentes: Hacia la profesionalización docente con TIC. Profesorado: Revista de Currículum y Formación del Profesorado, 22(1), 41-67. https://recyt.fecyt.es/index.php/profesorado/article/view/63620/

Torrecillas, T., Vázquez, T., Suárez, R. y Fernández, L. M. (2020). El papel de los padres en el comportamiento online de menores hiperconectados. Revista Latina de Comunicación Social, 75, 121-148. https://www.doi.org/10.4185/RLCS-2020-1419

UNICEF (2020). Manifiesto de la infancia y adolescencia 2020. https://bit.ly/2NBwdRh

Wood, M. A., Bukowski, W. M. y Lis, E. (2016). The digital self: How social media serves as a setting that shapes youth's emotional experiences. Adolescent Research Review, 1(2), 163-173. https://doi.org/10.1007/s40894-015-0014-8 
RLCS, Revista Latina de Comunicación Social, 79, 133-154

[Investigación] DOI: 10.4185/RLCS-2021-1528| ISSN 1138-5820| Año 2021

Yang, C. C., Holden, S. M. y Carter, M. D. (2018). Social media social comparison of ability (but not opinion) predicts lower identity clarity: Identity processing style as a mediator. Journal of youth and adolescence, 47(10), 2114-2128. https://doi.org/10.1007/s10964-017-0801-6

\section{AUTOR/ES:}

\section{María José Hernández-Serrano}

Profesora Titular del Departamento de Teoría e Historia de la Educación de la Universidad de Salamanca (España). Investiga y publica en torno al análisis de los procesos educativos mediados por tecnologías y las competencias digitales emergentes, la gestión pedagógica de la información digital y la promoción de usos críticos y creativos de los social media y transmedia. Es miembro de investigación del Grupo GIPEP: Procesos, Espacios y Prácticas Educativas de la Universidad de Salamanca y miembro activo de varias Redes de investigación internacionales y de diversos Comités Científicos en revistas indexadas sobre Tecnologías Educativas y Educomunicación.

mjhs@usal.es

Índice H: 13

Orcid ID: https://orcid.org/0000-0003-3818-993X

Google Scholar: https://scholar.google.com/citations?user=09R5STsAAAAJ\&hl=es

ResearchGate: https://www.researchgate.net/profile/Maria_Jose_Hernandez_Serrano

Scopus ID: https://www.scopus.com/authid/detail.uri?authorId=26656660100

Academia.edu:

https://usal.academia.edu/MAR\%C3\%8DAJOS\%C3\%89HERN\%C3\%81NDEZSERRANO

Publons: https://publons.com/researcher/2487221/maria-jose-hernandez-serrano/

\section{Paula Renés-Arellano}

Profesora Contratada Doctora de la Universidad de Cantabria (España). Actualmente imparte docencia en el Departamento de Educación en el área de Teoría de la Educación. Las líneas de investigación están vinculadas a las TIC, la alfabetización mediática y los valores. Estancias de investigación en diferentes universidades nacionales y extranjeras y revisora de diversas revistas científicas indexadas. Miembro del equipo de dirección del proyecto Educlips (https://www.educlips.es/). Coordinadora de España de la Red Interuniversitaria Euroamericana de Investigación sobre Competencia Mediática para la Ciudadanía (ALFAMED).

renesp.@unican.es

\section{Índice H: 14}

Orcid ID: https://orcid.org/0000-0003-0932-7694

Google Scholar: https://scholar.google.com/citations?user=3TDuh-oAAAAJ

ResearchGate: https://www.researchgate.net/profile/Paula-Renes-2

Scopus ID: https://www.scopus.com/authid/detail.uri?authorId=56041268700

Academia.edu: https://unican.academia.edu/PaulaRen\%C3\%A9sArellano

Publons: https://publons.com/researcher/2490929/paula-renes-arellano/

\section{Rosalynn Argelia Campos Ortuño}

Doctora en Educación, profesora asociada del Departamento de Teoría e Historia de la Educación en la Facultad de Educación de la Universidad de Salamanca, de la Universidad Internacional de la Rioja y Nebrija (España). Formación pedagógica con enfoque hacia el diseño curricular y la didáctica a través, en gestión de proyectos y de la calidad educativa para diferentes niveles académicos; y experta en TIC aplicadas a la educación. Es miembro del Grupo GIPEP: Procesos, Espacios y Prácticas Educativas y el Grupo MITA de la Universidad de Salamanca. Actualmente 
tiene abiertas líneas de investigación relacionadas con el uso de los dispositivos móviles y la identidad de los adolescentes, Recursos educativos en Abierto, y Robótica y programación educativa. rosecampos@usal.es

\section{Índice $\mathrm{H:} 7$}

Orcid ID: https://orcid.org/0000-0002-1456-753X

Google Scholar: https://scholar.google.es/citations?user=HYa9c4cAAAAJ\&hl=es

ResearchGate: https://www.researchgate.net/profile/Rosalynn-Campos

Scopus ID: $\underline{\text { https://www.scopus.com/authid/detail.uri?authorId=57217081899 }}$

Linkedin: https://es.linkedin.com/in/rosalynn-campos

Academia.edu: https://usal.academia.edu/ROSALYNNARGELIACAMPOSORTU\%C3\%910

\section{Belén González-Larrea}

Doctoranda de la Universidad de Salamanca -USAL (España), Magíster en Pedagogía Social (USAL), Psicología de la Educación de la Pontificia Universidad Católica del Ecuador - PUCE. Las líneas de investigación están vinculadas a Social Media, identidad digital, influencers y su influencia en los jóvenes, uso de Internet, impacto neuropsicológico, psicológico y socioeducativo. Cofundadora de NeuroClass, plataforma digital que se centró en la divulgación científica de la Neurociencia, Psicología y Educación.

Orcid ID: https://orcid.org/0000-0003-2983-081Xç

Google Scholar: https://scholar.google.com/citations?hl=es\&user=FUajaI0AAAAJ

ResearchGate: https://www.researchgate.net/profile/Belen-Gonzalez-Larrea

Publons:_ https://publons.com/researcher/4459715/belen-gonzalez/ 